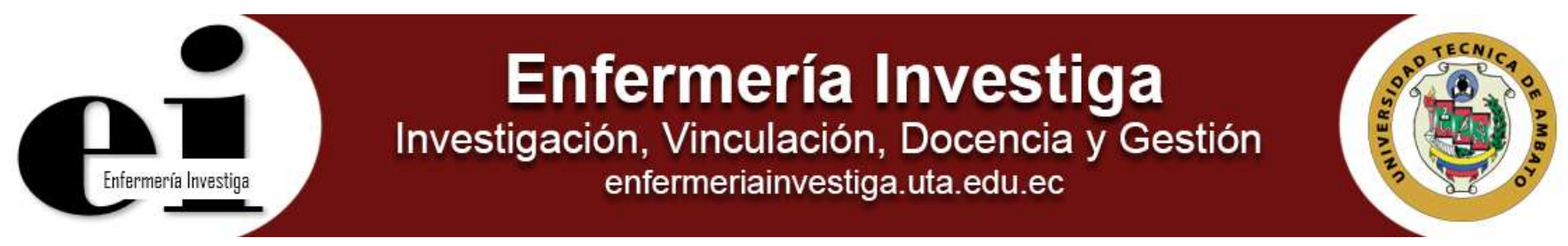

Artículo original

\title{
Calidad de la visita preoperatoria de enfermería
}

Quality of the preoperative nursing visit

José Luis Herrera López ${ }^{1}$, Eulalia Isabel Analuisa Jiménez ${ }^{1}$, Sara Verónica Guadalupe Núñez¹, Miguel Ángel de la Fuente Briz ${ }^{1}$, Alexandra Laguapillo Vergara ${ }^{1}$, Carmen de las Mercedes Cevallos Méndez ${ }^{1}$, Diana Nancy Martínez García $^{1}$, Fabiola Beatriz Chasillacta Amores ${ }^{1}$

\footnotetext{
${ }^{1}$ Carrera de Enfermería, Facultad Ciencias de la Salud, Universidad Técnica de Ambato, Ambato, Ecuador.
}

2477-9172 / 2550-6692 Derechos Reservados @ 2017 Universidad Técnica de Ambato, Carrera de Enfermería. Este es un artículo de acceso abierto distribuido bajo los términos de la Licencia Creative Commons, que permite uso ilimitado, distribución y reproducción en cualquier medio, siempre que la obra original es debidamente citada.

\section{Historia:}

Recibido: 28 abril 2017

Revisado: 06 mayo 2017

Aceptado: 11 mayo 2017

Palabras Claves: Enfermería Médico-Quirúrgica; visita preoperatoria; ansiedad; atención de enfermería

Keywords: Medical-Surgical Nursing; preoperative visit; anxiety; nursing care

\section{Resumen}

Introducción: La valoración del paciente es el primer paso en el proceso de enfermería, siendo necesaria para identificar e implementar las intervenciones que deben realizarse, valorando la eficacia de las mismas y minimizando los riesgos que conlleva.

Objetivo: Determinar de calidad de la visita preoperatoria de enfermería en el Hospital Municipal Nuestra Señora de la Merced, de la ciudad de Ambato, Ecuador.

Métodos: Se realizó un estudio observacional, descriptivo y longitudinal en todos los pacientes que fueron sometidos a cirugía electiva en el Hospital Municipal Nuestra Señora de la Merced $(n=80)$ así como al personal de enfermería que laboró en el área quirúrgica $(n=24)$. La investigación se desarrolló en el período comprendido entre enero a junio del año 2015. Se aplicó un cuestionario tanto a pacientes como al personal de enfermería que respondió a los objetivos de la investigación.

Resultados: Más de la mitad de los pacientes no fueron llamados por su nombre de forma constante $(n=48,60 \%)$. La información sobre los cuidados a realizar, no fue brindada en el $53,8 \%$ de los pacientes. Se evidenció la toma de signos vitales al ingreso del paciente como una práctica habitual $(n=23,95.8 \%)$.

Conclusiones: Se evidenció una atención de enfermería en cierta medida rutinaria, que no brindó la información necesaria a los pacientes en relación a su condición quirúrgica. La visita preoperatoria presentó dificultades en cuanto a la relación enfermero-paciente, la preparación de la piel previa a la cirugía y la instrucción a pacientes sobre los ejercicios respiratorios.

\section{Abstract}

Introduction: The assessment of the patient is the first step in the nursing process, being necessary to identify and implement the interventions that must be performed, assessing the effectiveness of the same and minimizing the risks entailed.

Objetive: To determine the quality of the preoperative nursing visit at the Nuestra Señora de la Merced Municipal Hospital, in the city of Ambato, Ecuador.

Methods: An observational, descriptive, and longitudinal study was performed in all patients who underwent elective surgery at the Nuestra Señora de la Merced Municipal Hospital $(n=80)$ as well as the nursing staff who worked in the surgical area $(n=24)$. The research was carried out between january and june of the year 2015. A questionnaire was applied to both patients and nursing staff who responded to the research objectives.

Results: More than half of the patients were not called by their name $(n=48,60 \%)$. The information on the care to be performed was not provided in $53.8 \%$ of the patients. It was evidenced the taking of vital signs to the patient's entrance as a habitual practice $(n=23,95.8 \%)$.

Conclusions: Nursing care was evidenced to some extent routine, which did not provide the necessary information to the patients in relation to their surgical condition. The preoperative visit presented difficulties regarding the nurse-patient relationship, preparation of the skin prior to surgery and instruction to patients about breathing exercises.

Autor de correspondencia:

José Luis Herrera López. Carrera de Enfermería, Facultad Ciencias de la Salud, Universidad Técnica de Ambato, Ambato, Ecuador. E-mail: j.I.h.lopez@hotmail.com 


\section{Introducción}

La Enfermería Quirúrgica abarca el área quirúrgica por medio del fomento de la salud, la prevención de la enfermedad y los cuidados a pacientes con alguna condición quirúrgica ${ }^{1}$. Cada intervención quirúrgica origina en el paciente una importante alteración fisiológica y emocional. Esta vertiente de la enfermería abarca los cuidados, la convivencia, la disciplina y la técnica; como elementos necesarios para ofrecer una asistencia satisfactoria al paciente ${ }^{2,3}$.

La valoración del paciente es el primer paso en el proceso de enfermería, siendo necesaria para identificar e implementar las intervenciones que deben realizarse, valorando la eficacia de las mismas y minimizando los riesgos que conlleva ${ }^{4}$. Esta valoración comprende la información de cualquier índole sobre la situación y la evolución de un paciente a lo largo del proceso asistencial, como parte de la historia clínica de enfermería ${ }^{5}$; y la adecuada confección de esta permite la detección de patologías preexistentes en el $97 \%$ de los casos, así como la toma de decisiones posteriores referentes a la necesidad de estudios complementarias, interconsultas y cuidados perioperatorios ${ }^{6}$.

Como parte del proceso quirúrgico, los diagnósticos enfermeros más frecuente son la ansiedad o temor relacionado con la intervención quirúrgica, la anestesia o los resultados impredecibles que estas puedan desencadenar ${ }^{4,6}$. Los daños a la identidad personal, así como las amenazas a la integridad física y al autosistema, figuran entre los desencadenantes de la ansiedad en el proceso quirúrgico. Es la ansiedad una experiencia individual que no puede objetivarse de forma directa, provocada por lo desconocido y precediendo a todas las nuevas experiencias?

La visita preoperatoria es un instrumento al servicio de enfermería como parte del proceso de atención al paciente quirúrgico, con énfasis en la colaboración entre el personal de la unidad de hospitalización y el del bloque quirúrgico ${ }^{6}$. Puede definirse como el proceso por medio del cual se personalizan los cuidados de enfermería previo a una cirugía electiva, por medio de una entrevista personalizada que persigue informar al paciente sobre el autocuidado, así como su asesoramiento y disminución de la ansiedad y el temor $^{7}$. Entre la información útil para reducir la ansiedad en el paciente quirúrgico está la referente al procedimiento a realizar, el comportamiento sobre lo que se debe hacer antes, durante y después del procedimiento y las sensaciones que puede experimentar el paciente ${ }^{8}$.

El presente artículo tuvo como finalidad determinar de calidad de la visita preoperatoria de enfermería en el Hospital Municipal Nuestra Señora de la Merced, de la ciudad de Ambato, Ecuador.

\section{Materiales y Métodos}

Se realizó un estudio observacional, descriptivo y longitudinal en todos los pacientes que fueron sometidos a cirugía electiva en el Hospital Municipal Nuestra Señora de la Merced $(n=80)$ así como al personal de enfermería que laboró en el área quirúrgica $(n=24)$. Se solicitó su conformidad en participar del estudio, no hubo casos donde el estado físico, mental o intelectual impidiese dicho consentimiento.

La investigación se desarrolló en el período comprendido entre enero a junio del año 2015. Se aplicó un cuestionario tanto a pacientes como al personal de enfermería que respondió a los objetivos de la investigación.

La frecuencia de los aspectos valorados en el cuestionario fue relacionada de la siguiente forma:

- Siempre: En la totalidad de las veces.

- Casi siempre: En más de la mitad de las veces.

- Algunas veces: En menos de la mitad de las veces.

- Nunca: En ninguna ocasión.

Se confeccionó una base de datos en Microsoft SPSS 20.0. Como medidas de resumen fuero utilizadas frecuencias absolutas y relativas porcentual.

La investigación siguió los principios y recomendaciones para una investigación biomédica en seres humanos, adhiriéndose a los convenios internacionales vigentes en cumplimiento de los principios éticos, explicando con la finalidad de su comprensión para el consentimiento informado, las características de la investigación y la trascendencia que sus resultados tendrían en beneficio de la población estudiada.

\section{Resultados}

Se encontró que más de la mitad de los pacientes no fueron llamados por su nombre de forma constante $(n=48,60 \%)$. Un aspecto tan importante en la etapa preoperatoria, como lo es la información sobre los cuidados a realizar, no fue brindado en el $53,8 \%$ de los pacientes. De igual forma no se brindó información sobre la cirugía a realizarse y los pacientes no percibieron trasmisión de seguridad en la mayoría de las veces (tabla 1).

Entre los aspectos encuestados al personal de enfermería, como parte de la vivista preoperatoria, se constató dificultades en cuanto a la presentación del personal por su nombre, la preparación de la piel acorde al tipo de cirugía a realizarse y la explicación de los ejercicios respiratorios en el paciente. Como aspectos favorables, se evidenció el registro de los signos vitales al ingreso del paciente con cierta regularidad, así como la verificación del ayuno previo a la cirugía (tabla 2).

Tabla 1. Atención de enfermería según pacientes, Hospital Municipal Nuestra Señora de la Merced, enero a junio de 2015.

\begin{tabular}{lcccc}
\hline Frecuencia/Criterio & $\begin{array}{c}\text { Llamado por su } \\
\text { nombre } \mathbf{n}(\%)\end{array}$ & $\begin{array}{c}\text { Información sobre } \\
\text { cuidados a realizar } \\
\mathbf{n}(\%)\end{array}$ & $\begin{array}{c}\text { Información sobre } \\
\text { cirugía a realizarse } \\
\mathbf{n}(\%)\end{array}$ & $\begin{array}{c}\text { Trasmisión de } \\
\text { seguridad hacia el } \\
\text { paciente } \mathbf{n}(\%)\end{array}$ \\
\hline Siempre & $15(18.8)$ & $5(6.3)$ & $1(1.3)$ & $24(30.0)$ \\
Casi siempre & $17(21.3)$ & $12(15.0)$ & $5(6.3)$ & $34(42.5)$ \\
Algunas veces & $30(37.5)$ & $20(25.0)$ & $10(12.5)$ & $10(12.5)$ \\
Nunca & $18(22.5)$ & $43(53.8)$ & $64(80.0)$ & $12(15.0)$ \\
\hline Fuente:
\end{tabular}

Fuente: Cuestionario aplicado a pacientes, Hospital Municipal Nuestra Señora de la Merced. 
Tabla 2. Visita preoperatoria según personal de enfermería, Hospital Municipal Nuestra Señora de la Merced, enero a junio de 2015.

\begin{tabular}{lcccccc}
\hline Frecuencia/Criterio & $\begin{array}{c}\text { Presentación } \\
\text { ante el } \\
\text { paciente } \mathrm{n}(\%)\end{array}$ & $\begin{array}{c}\text { Información } \\
\text { sobre cirugía } \\
\text { a realizarse } \\
\mathbf{n}(\%)\end{array}$ & $\begin{array}{c}\text { Registro } \\
\text { de signos } \\
\text { vitales } \\
\mathbf{n}(\%)\end{array}$ & $\begin{array}{c}\text { Preparación } \\
\text { de la piel } \mathbf{n}(\%)\end{array}$ & $\begin{array}{c}\text { Verificación } \\
\text { del ayuno } \\
\mathbf{n}(\%)\end{array}$ & $\begin{array}{c}\text { Ejercicios } \\
\text { respiratorios } \\
\mathbf{n}(\%)\end{array}$ \\
\hline Siempre & $1(4.2)$ & - & $3(12.5)$ & $2(8.3)$ & $18(75.0)$ & $4(16.7)$ \\
Casi siempre & $2(8.3)$ & $3(12.5)$ & $20(83.3)$ & $4(16.7)$ & $5(20.8)$ & - \\
Algunas veces & $9(37.5)$ & $13(54.2)$ & - & $7(29.2)$ & - & $10(41.7)$ \\
Nunca & $12(50.0)$ & $8(33.3)$ & $1(4.2)$ & $11(45.8)$ & $1(4.2)$ & $10(41.7)$ \\
\hline
\end{tabular}

Fuente: Cuestionario aplicado al personal de enfermería, Hospital Municipal Nuestra Señora de la Merced.

\section{Discusión}

Según refirieron los pacientes, el personal de enfermería no suele llamarlos por su nombre asiduamente $(n=48,60 \%)$, por lo cual se incumple un derecho fundamental del paciente, coincidiendo con reportes de otros autores ${ }^{9}$. Como parte de la amabilidad y el buen trato, el llamado por su nombre ha sido identificado como el aspecto más valorado por los pacientes en estudios similares ${ }^{10}$.

Más de la mitad de los pacientes apuntaron que nunca se les brindó información acerca de los cuidados de enfermería a realizarse. Estos resultados fueron insatisfactorios, si se tiene en cuenta que la enfermería comprende el suministro de información necesaria al paciente, de manera que cubra sus necesidades, mejore su autoayuda o mitigue el sufrimiento $^{11}$. Al respecto se ha considerado una apuesta en favor de la autonomía de las personas, de forma que sea el propio individuo capaz y libre, quien decida si se somete o no a un proceder o cuidado en particular ${ }^{12}$.

Referente a la cirugía a realizarse, la mayoría de los pacientes nunca recibieron información al respecto $(n=64$, 80\%). Al respecto la Organización Mundial de la Salud (OMS) ha señalado que: "Enfermería confirmará verbalmente con el paciente su identidad, el tipo de intervención prevista, el lugar anatómico de la intervención y el consentimiento del paciente para su realización. Aunque pueda parecer repetitivo, este punto es esencial para garantizar que no se opera a la persona equivocada o el lugar anatómico equivocado, ni se realiza una intervención equivocada"13

La trasmisión de seguridad al paciente es un aspecto clave para tratar la ansiedad del paciente durante el preoperatorio ${ }^{8}$, toda vez que la reacción ansiosa de un paciente al experimentar una cirugía es uno de los mayores factores que afectan los ajustes perioperatorios ${ }^{14}$. Un aspecto favorable en el presente estudio fue la trasmisión de seguridad al paciente en la mayoría de veces.

A través del cuestionario aplicado a los profesionales de enfermería, se constató que el $50 \%$ de estos nunca se presentó por su nombre a los pacientes. En este sentido se puede señalar que una relación enfermero-paciente de forma fluida contribuye considerablemente a disminuir la ansiedad preoperatoria, otros autores han encontrado una mejor identificación por parte del personal de enfermería ${ }^{9,15}$.

Más de la mitad de los enfermeros no brindaron información a sus pacientes sobre cirugía a realizarse, teniendo en cuenta la naturaleza educativa de la enfermería ${ }^{16}$. Un estudio realizado en España sobre la satisfacción del paciente en el tratamiento quirúrgico programado del cáncer colorrectal, arrojó un $95.4 \%$ de pacientes que consideraron suficiente la información recibida ${ }^{17}$. Una investigación similar realizada en Perú, evidenció una deficiente información a pacientes sobre el proceder quirúrgico a practicarse $(26 \%)^{18}$.
Los signos vitales constituyen una herramienta valiosa, como indicadores del estado funcional de los pacientes sin embargo, estos deben ser interpretados oportunamente en el contexto clínico ${ }^{19}$. En el estudio realizado se evidenció la toma de signos vitales al ingreso del paciente como una práctica habitual $(n=23,95.8 \%)$, resultados superiores a los obtenidos en un estudio sobre el manejo y administración de hemoderivados por el personal de enfermería, donde fueron registrados los signos vitales en un $91.8 \%$ de los pacientes $^{20}$.

La preparación de la piel previa a la cirugía resulta un factor clave en la prevención de la infección del sitio quirúrgico (ISQ), toda vez que inevitablemente la flora endógena contaminará dicho sitio. Algunos reportes señalan la disminución de colonias bacterianas en la piel asociada al baño preoperatorio del enfermo con jabón antiséptico, sin embargo, no existe evidencia sólida al respecto ${ }^{21}$. Previa a la incisión quirúrgica es obligatorio preparar la piel con algún antiséptico como puede ser el iodopovidona, alcohol o clorhexidina, después de haber limpiado las áreas visiblemente sucias ${ }^{22}$. Resulta preocupante el hecho que casi la mitad del personal de enfermería refirió no realizar esta preparación, con lo cual podría incrementarse la incidencia de ISQ de forma considerable en Hospital Municipal Nuestra Señora de la Merced.

La verificación del ayuno como parte de la evaluación preoperatoria, contribuye a la disminución de las complicaciones durante el preoperatorio ${ }^{23}$. En el presente estudió se obtuvo un $75 \%$ de verificación del ayuno por parte del personal de enfermería en todos los casos, a la vez que un $20.8 \%$ de este personal refirió verificarlo en más de la mitad de las veces. Esta es una práctica de rutina en la mayoría de las unidades quirúrgicas, toda vez que se han reportado hospitales donde no es tenida en cuenta como parte de la evaluación preoperatoria del paciente ${ }^{24}$.

En la visita preoperatoria se constató que el $41.7 \%$ de los enfermeros no instruyeron a los pacientes acerca de los ejercicios respiratorios para el posquirúrgico, siendo estos de vital importancia tanto previo a la cirugía como después a esta; otros autores han reportado hasta un $72.9 \%$ de esta práctica previa a la cirugía ${ }^{25}$.

\section{Conclusiones}

Acorde a la percepción de los pacientes, la calidad de la visita preoperatoria que se brindó en el Hospital Municipal Nuestra Señora de la Merced fue susceptible de mejoras. Se evidenció una atención de enfermería en cierta medida rutinaria, que no brindó la información necesaria a los pacientes en relación a su condición quirúrgica, toda vez que este es un elemento determinante para disminuir la ansiedad de estos. Desde el punto de vista técnico, la visita preoperatoria presentó dificultades en cuanto a la relación enfermero-paciente, a lo que se sumó la no preparación de la piel previa a la cirugía y la no instrucción a pacientes sobre los ejercicios respiratorios. 


\section{Referencias}

1. Bernalte-Martí V, Orts-Cortés MI, Maciá-Soler L. Percepción de los profesionales de enfermería y auxiliares de enfermería sobre cultura de seguridad del paciente en el área quirúrgica. Enferm Clínica [en línea]. marzo de 2015 [citado 5 de junio de 2017];25(2):64-72. Disponible

http://linkinghub.elsevier.com/retrieve/pii/S1130862114001223

2. Rubio LC, Ruiz JRC, Rebaza LC. Calidad del cuidado de enfermería en la seguridad integral del paciente quirúrgico del hospital base Víctor Lazarte Echegaray, Trujillo-2014. SCIÉNDO [en línea]. 31 de diciembre de 2015 [citado 5 de junio de 2017];17(1). Disponible en: http://revistas.unitru.edu.pe/index.php/SCIENDO/article/view/1026

3. Herrera RO, Rodríguez ALH, Peña YPB, Portelles LR, Valverde RAR. Intervención educativa en la carrera de Enfermería, como estrategia curricular de Medicina Tradicional y Natural. Rev Electrónica Dr Zoilo E Mar Vidaurreta [en línea]. 17 de diciembre de 2015 [citado 5 de junio de 2017];38(2). Disponible http://revzoilomarinello.sld.cu/index.php/zmv/article/view/564

4. Gonçalves TF, de Medeiros VCC. A visita pré-operatória como fator atenuante da ansiedade em pacientes cirúrgicos. Rev SOBECC [en línea]. 2016 [citado 5 de junio de 2017];21(1):22-27. Disponible en: http://files.bvs.br/upload/S/1414-4425/2016/v21n1/a5570.pdf

5. Borja A, Michelle K. Evaluación de la intervención de enfermería en el preoperatorio inmediato en pacientes atendidos en el servicio de emergencia del hospital un canto a la vida, durante el mes de agosto a diciembre del 2016. 2017 [citado 5 de junio de 2017]; Disponible en: http://dspace.udla.edu.ec/handle/33000/6803

6. González González JM. Efectividad de una intervención enfermera para la adaptación quirúrgica del paciente hipertenso. NURE Investig [en línea]. 2015; Disponible en: http//www nureinvestigacion es/OJS/index.php/nure/article/view/89

7. Miguel Romeo MC, Sagardoy Muniesa L. Efectos del uso de un tríptico informativo en la reducción de la ansiedad y el dolor perioperatorios en pacientes intervenidos de patología urológica. Enferm Clínica [en línea]. julio de 2014 [citado 5 de junio de 2017];24(4):233-40. Disponible

http://linkinghub.elsevier.com/retrieve/pii/S1130862113001885

8. Svensson M, Nilsson U, Svantesson M. Patients' experience of mood while waiting for day surgery. J Clin Nurs [en línea]. 1 de septiembre de 2016 [citado 5 de junio de 2017];25(17-18):2600-8. Disponible en: http://onlinelibrary.wiley.com/doi/10.1111/jocn.13304/abstract

9. Angulo AMA. Calidad del cuidado enfermero en la dimensión interpersonal del paciente hospitalizado en el Hospital Edgardo Rebagliati Martins. Rev Peru Obstet Enferm [en línea]. 13 de septiembre de 2016 [citado 8 de junio de 2017];7(2). Disponible en: http://www.aulavirtualusmp.pe/ojs/index.php/rpoe/article/view/662

10. Pangrazzi A. Girasoles junto a sauces: en diálogo con los enfermos. Editorial SAL TERRAE; 2002. $234 \mathrm{p}$.

11. Vargas-Toloza RE. Cuidado humanizado al paciente críticamente enfermo: enfermería pieza clave en la atención. Cienc Cuid [en línea]. 3 de abril de 2017 [citado 8 de junio de 2017];4(1):21-7. Disponible en: http://revistas.ufps.edu.co/ojs/index.php/cienciaycuidado/article/view/ 918

12. García Millán Á. La información al paciente como pieza clave de la calidad asistencial. Rev Clínica Med Fam. 2009;2(6):275-9.

13. UNICEF. Estado mundial de la infancia 2005: la infancia amenazada. UNICEF; 2004. $164 \mathrm{p}$

14. Carapia-Sadurni A, Mejía-Terrazas GE, Nacif-Gobera L, HernándezOrdóñez MN. Efecto de la intervención psicológica sobre la ansiedad preoperatoria. Rev Mex Anestesiol [en línea]. 2011 [citado 8 de junio
2017];34(4):260-263.

Disponible

http://www.medigraphic.com/pdfs/rma/cma-2011/cma114e.pdf

15. Romero Ameri LC. Percepción del paciente acerca de la calidad de atención que brinda la enfermera en el servicio de medicina en el Hospital Nacional Daniel Alcides Carrión. 2008 [citado 8 de junio de 2017]; Disponible

http://cybertesis.unmsm.edu.pe/handle/cybertesis/482

16. Urra M E, Jana A A, García V M. Algunos aspectos esenciales del pensamiento de Jean Watson y su teoría de Cuidados Transpersonales. Cienc Enferm [en línea]. diciembre de 2011 [citado 8 de junio de 2017];17(3):11-22. Disponible en: http://www.scielo.cl/scielo.php?script=sci abstract\&pid=S0717http://wWW.scielo.cl/scielo.php?script=sci_abstract\&

17. Rodríguez-Cuéllar E, Ruiz-López $P$, Alcalde-Escribano J, LandaGarcía I, Villeta-Plaza R, Jaurrieta-Mas E. Satisfacción del paciente tras el tratamiento quirúrgico del cáncer colorrectal. Cir Esp [en línea]. 1 de enero de 2004 [citado 8 de junio de 2017];76(4):237-45. Disponible

en: http://www.sciencedirect.com/science/article/pii/S0009739X04789745

18. Ninantay Q, María A. Opinión que tienen los pacientes del Servicio de Medicina General sobre la calidad de atención de la enfermera en el Hospital Nacional Arzobispo Loayza. Univ Nac Mayor San Marcos [en línea]. 2005 [citado 8 de junio de 2017]; Disponible en: http://cybertesis.unmsm.edu.pe/handle/cybertesis/1006

19. Jaimes MEF, Grajales RAZ, Cervantes JMO, Antonio MTR. La evaluación de la calidad de los signos vitales como indicador de proceso en la Gestión del Cuidado de Enfermería. Rev Mex Enferm Cardiológica [en línea]. 2010 [citado 10 de junio de 2017];18(3):65-70. Disponible en: http://www.medigraphic.com/pdfs/enfe/en2010/en103d.pdf

20. Flores-Torrecillas R, Carballo-Monreal MR, Alvarez-Villaseñor AS, Valdez-Márquez ML, González-Ojeda A, Fuentes-Orozco C. Manejo y administración de hemoderivados por personal de enfermería en un hospital de segundo nivel. Enferm Univ [en línea]. julio de 2014 [citado 10 de junio de 2017];11(3):94-100. Disponible en: 10 de junio de 2017];11(3):94-100. Disponible en:
http://www.sciencedirect.com/science/article/pii/S1665706314726727

21. Compte DV, Pineda EBG, Hernández ESS, Castillejos A. Infecciones de sitio quirúrgico. De la patogénesis a la prevención. Enfermedades Infecc Microbiol [en línea]. 2008 [citado 10 de junio de 2017];28(1):24. Disponible en: http://www.medigraphic.com/pdfs/micro/ei2008/ei081e.pdf

22. Vargas-Domínguez A, Ortega-León LH, Rodríguez-Báez A, LópezLópez JM, Zaldívar-Ramírez FR, Montalvo-Javé E. Vigilancia epidemiológica de infección del sitio operatorio superficial. Estudio comparativo de tres años. Cir Cir [en línea]. 2001 [citado 10 de junio de 2017];69(4):177-180. Disponible en: http://www.medigraphic.com/pdfs/circir/cc-2001/cc014e.pdf

23. Víctor Troncoso C. Evaluación preoperatoria. Rev Médica Clínica Las Condes [en línea]. mayo de 2011 [citado 10 de junio de 2017];22(3):340-9. Disponible en:

24. Walter Checa G. Estudio comparativo de protocolos de preparación prequirúrgica. 2013 [citado 10 de junio de 2017]; Disponible en: http://repositorio.ual.es/bitstream/handle/10835/2485/Trabajo.pdf?seq uence=1\&isAllowed=y

25. Balsera BC, Sánchez LM, Gómez ÓG, Moyano NG, Cabra PD, López RG. Descripción de un protocolo de fisioterapia respiratoria a pacientes sometidos a cirugía abdominal alta. Rev Fisioter [en línea]. 2013;12(2):45-52. Disponible

http://www.ucam.edu/sites/default/files/revista-

fisio/descripcion_de_un_protocolo_de_fisioterapia_respiratoria_a.pdf 